\title{
Dust physics in the nucleus of NGC 4151
}

\author{
K. Schnülle ${ }^{1}$, J.-U. Pott ${ }^{1}$, H.-W. Rix ${ }^{1}$, R. Decarli ${ }^{1}$, B. M. Peterson ${ }^{2}$, and W. Vacca ${ }^{3}$ \\ 1 Max Planck Institut für Astronomie, Königstuhl 17, 69117 Heidelberg, Germany \\ e-mail: schnuelle@mpia.de \\ 2 Department of Astronomy, The Ohio State University, $140 \mathrm{~W}$ 18th Ave, Columbus, OH 43210, USA \\ 3 SOFIA-University Space Research Association, NASA Ames Research Center, Mail Stop N211-3, Moffett Field, CA 94035, USA
}

Received 30 April 2013 / Accepted 22 July 2013

\section{ABSTRACT}

\begin{abstract}
The unified model of active galactic nuclei (AGNs) presumes the existence of a so-called dusty torus around the outer edge of the broad-line region. Despite the solid observational evidence for the existence of dust around AGNs and our growing information on the characteristic scales of these obscuring tori, the origin of this dust and its morphology are not yet well understood.

Using dust reverberation mapping, we monitor the effects of AGN continuum variability to determine the temperature and covering factor of the circumnuclear dust, in order to constrain the physical conditions for dust survival and formation in the radiation field of the AGN. Multi-band photometry observations in the $z, Y, J, H$, and $K$ bands were carried out on the nucleus of the prototypical Seyfert 1 galaxy NGC 4151 over six epochs from 2010 January to June, supported by spectroscopic observations, in order to investigate the response of the hot dust to varying accretion disk emission.

Our data confirm that most of the hot dust reacts to increased radiation from the central source with a delayed brightening of $\sim 50$ days. In accretion disk brightening, we see no signatures of dust destruction in our data. The innermost dust appears to increase in temperature rather than sublimate, suggesting that it is cooler than sublimation temperature and located beyond the current sublimation radius. We characterize the dust geometry by interpreting the wavelength-dependent reverberation response with a simplified torus model, pointing to a static radially extended distribution of the central $(\sim 0.1 \mathrm{pc})$ hot dust.
\end{abstract}

Key words. galaxies: active - galaxies: nuclei - galaxies: Seyfert - infrared: galaxies - galaxies: individual: NGC 4151

\section{Introduction}

Dust is a cornerstone of the unified AGN model, positing that type 1 and type 2 AGNs are intrinsically the same phenomenon, and attributing their different spectral energy distributions (SEDs) to the existence of an optically thick, non-spherically symmetric, dusty structure located around the broad-line region (BLR). This so-called dust torus is assumed to obscure the central region of type 2 AGNs, resulting in a different SED and consequently resolving the apparent dichotomy between type 1 and type 2 AGNs (Antonucci 1993; Urry \& Padovani 1995).

Over the past years, convincing observational evidence has indicated that hot dust resides at the outer edge of the BLR (Barvainis 1987; Heisler et al. 1997; Osterbrock \& Ferland 2006), and with the rise of infrared interferometry, it has finally become feasible to directly resolve the near-infrared (NIR) hot dust around nearby Seyfert 1 AGNs (Swain et al. 2003; Weigelt et al. 2012). The observational data were essential in breaking degeneracies in radiative transfer models, resulting in the current overall picture: a clumpy distribution of dust clouds that accumulate along the equatorial plane and constitute the shape of a torus.

Despite this substantial increase in our knowledge of dust in AGNs, its origin and detailed morphology are not yet identified. Self-consistent AGN torus models (Krolik \& Begelman 1988; Schartmann et al. 2010) assume that dust is brought from outside to the central nuclear region, until it sublimates in the radiation field of the AGN. This picture, in which the innermost hot dust accumulates at the dust sublimation radius, is consistent with the $V-K$ reverberation measurements of Koshida et al. (2009), who reported a strongly varying location of the innermost dust in the Seyfert 1 galaxy NGC 4151. In intrinsic dust formation scenarios (Elvis et al. 2002; Elitzur \& Shlosman 2006), in contrast, the dust probably forms well outside the sublimation radius, from the outflows of accretion disk (AD) winds. This is supported by interferometric observations of Pott et al. (2010), who found no change in the location of the hot $K$-band dust in NGC 4151 in response to varying AD brightness.

We present here the first results on our AGN hot dust reverberation program, in which we monitor seven nearby, bright and variable Seyfert 1 galaxies from the optical to the NIR. Specifically, we use dust reverberation mapping of NGC 4151 as a tool to study in detail the temperature evolution and radial profile of the hot dust around AGNs. The galaxy NGC 4151, one of the brightest and most nearby Seyfert 1 galaxies on the sky and highly variable in its continuum emission, appears to be a perfect candidate for this purpose.

After describing the observations and data reduction (Sect. 2), we introduce the methods used for analyzing the data in Sect. 3. Our current results on NGC 4151 are presented in Sect. 4, followed by discussion in Sect. 5. We summarize our results and give a brief outlook on the project in Sect. 6 .

\section{Observations and data reduction}

To start our AGN hot dust monitoring program, we observed NGC 4151 from 2010 January - June with roughly monthly sampling, using NIR multi-band photometry obtained with Omega 2000 (Kovács et al. 2004), a NIR wide-field camera mounted on the $3.5 \mathrm{~m}$ telescope in Calar Alto, Spain ${ }^{1}$. The field of view

1 Based on observations collected at the Centro Astronómico Hispano Alemán (CAHA), operated jointly by the Max-Planck Institut für Astronomie and the Instituto de Astrofisica de Andalucia (CSIC). 
of Omega 2000 is 15 ' $^{\prime} \times 15$ '.4, and its $2048 \times 2048$ pixel detector has a scale of 0.45 pixel $^{-1}$. We obtained broadband photometry of NGC 4151 and three reference stars in the field of view in the spectral bands $z, Y, J, H$, and $K$, in six epochs in 2010. Dithering was used to estimate the sky background. The weather conditions ranged from clear to photometric, and the seeing was $\sim 0.9^{\prime \prime}-1.5^{\prime \prime}$. We reduced the images using IRAF. After dark subtraction, we performed flat-fielding on each frame using sky flats. Then we subtracted an additional sky image, obtained from the dithered target frames.

We calibrated the fluxes of our reference stars with the 2MASS PSC (Skrutskie et al. 2006) fluxes for the $J H K$ bands. For the $z Y$ bands, we used a database containing model spectra of main sequence stars (kindly provided by R. van Boekel, priv. com.). For each reference star, we determined the best fit spectrum to the $B V J H K$ fluxes as listed by SIMBAD and calculated the $z Y$ photometry from that spectrum. The flux ratios between the reference stars were found to be temporally stable for each filter, with resulting photometric errors of $\sim 0.005-0.03 \mathrm{mag}$ (dominated by spatial variations of the point spread function (PSF)). For the nuclear flux calibration of NGC 4151, a conversion factor for each epoch and filter was calculated, using the mean of the corresponding calibration factors of the reference stars. To avoid host galaxy contributions, we then extracted the nuclear flux of NGC 4151 by performing a PSF-bulge-disk decomposition with GALFIT (Peng et al. 2002), with statistical errors of $\sim 0.01 \mathrm{mag}$.

In order to correct for emission line contributions in our data, we obtained a NIR spectrum of NGC 4151 on 2010 February 26 (LXD2.1, 2-5 $\mu \mathrm{m}$, slit size $0.3^{\prime \prime}$, slit angle $191^{\circ}$ ) and February 27 (SXD, 0.8-2.5 $\mu \mathrm{m}$, slit size 0.3", slit angle $188^{\circ}$ ), in parallel with the 2010 February photometry, using the SpeX spectrograph (Rayner et al. 2003) at the NASA Infrared Telescope Facility (IRTF), a $3 \mathrm{~m}$ telescope on Mauna Kea, Hawai'i. Conditions were clear and the seeing was $\sim 0.6^{\prime \prime}-0.7^{\prime \prime}$. The observations of NGC 4151 were followed by observations of an A0V star, used as a telluric and flux standard. The data were reduced using the IRTF software package Spextool (Cushing et al. 2004), and calibrated as described in Vacca et al. (2003). The SXD and LXD2.1 spectra were then combined to produce a final spectrum covering $0.8-5 \mu \mathrm{m}$. Our 2010 February relative photometry between the different passbands is in excellent agreement with the synthetic photometry derived from the IRTF spectrum (see Fig. 1). For each filter, we applied a correction factor to our data $(8 \%$ in $z Y, 15 \%$ in $J, 2 \%$ in $H K$ ), given by the ratio of the 2010 February photometry to the continuum flux level at the respective effective wavelength, to remove the emission line contributions. We use these photometric measurements throughout this Letter.

\section{Methods}

The circumnuclear dust absorbs the AD radiation and re-emits it in the infrared. Dust at a radial distance $R_{\text {dust }}$ from the AD responds to variations in the incident flux with a characteristic lag time $\tau=R_{\text {dust }} / c$, where $c$ is the speed of light. The dust emission can be approximated by the blackbody function

$F_{\lambda}(T, \Omega)=\Omega \frac{2 h c^{2}}{\lambda^{5}} \frac{1}{\mathrm{e}^{h c / \lambda k_{\mathrm{B}} T}-1}$,

and is thus determined by the dust blackbody temperature $T$ and the solid angle $\Omega$ that the emitting dust clouds subtend on the sky, as seen from Earth. This solid angle is directly proportional to the emitting blackbody surface area.

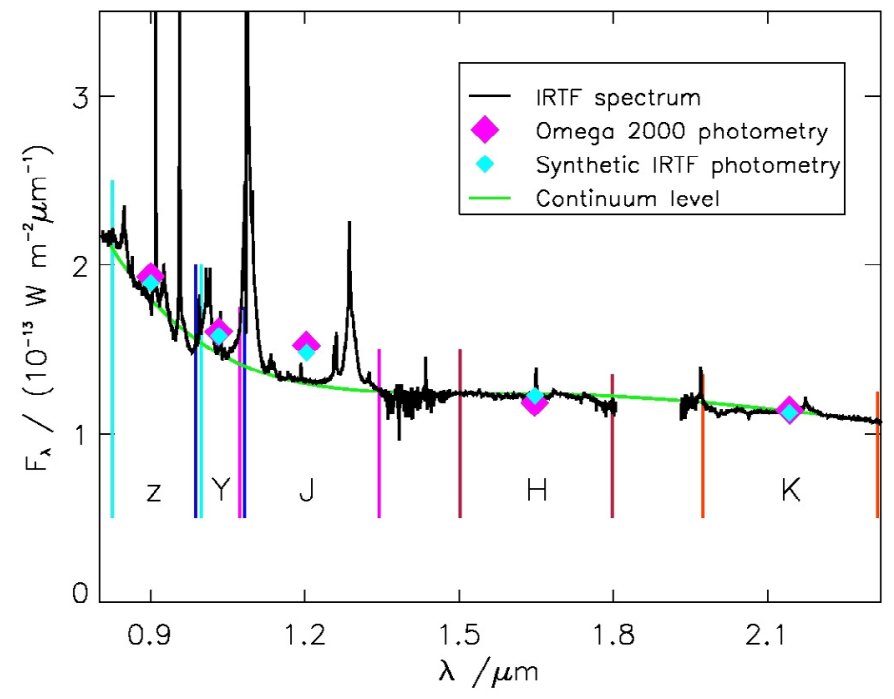

Fig. 1. Our 2010 February (MJD 55252) photometry and the IRTF spectrum of NGC 4151. Vertical lines mark the $z Y J H K$ filter widths.

By monitoring the hot dust signal as it responds to the AD signal, we determine the reverberation lag time and the evolution of the hot dust temperature and emitting surface, to measure the radial dust distribution and to infer whether or not the hot dust is close to sublimation. We note that the dust sublimation temperature depends on the assumed dust species, composition and grain sizes, all of which might change significantly with distance from the source. Without detailed knowledge of these dust properties, it is not possible to determine if the innermost dust is close to sublimation from measuring the dust temperature in one single epoch. Monitoring the temperature for an extended period allows us to draw more reliable conclusions about the immediate dust state, since, following increasing AD emission, we are then able to discriminate between a constant temperature (in case of sublimation) and rising temperatures (no sublimation).

To separate our $z Y J H K$ photometry into $\mathrm{AD}$ and hot dust contributions, we decomposed the nuclear fluxes according to a two-component model: a power-law of the form $C \lambda^{-\alpha}$ for the AD which dominates the $z Y$ bands, plus a blackbody function (Eq. (1)) for the hot dust dominating the $H K$ bands (Kobayashi et al. 1993; Riffel et al. 2009). Considering the mild AD flux variations in the observed period $(\approx 15 \%$, as traced by the $z$ band), it is a reasonable first order approximation to assume $\alpha=$ const. We fixed $\alpha=2.02$, corresponding to the mean value of $\alpha$ over the different epochs when it is a free parameter. This is consistent with Riffel et al. (2009), who found $\alpha=2.25$ for the same galaxy in a different epoch. We fitted this model to our SED using the IDL routine mpfit (for fit results see Table 1).

\section{Results}

The temporal evolution of the band-normalized photometry of the nucleus of NGC 4151 is shown in Fig. 2. We find that the flux variations in the other bands lag behind those in the $z$ band, which is dominated by $\mathrm{AD}$ emission, and the lag increases with wavelength. This band-dependent behavior from $z$ to $K$ shows increasing dust contribution and decreasing AD contribution going to longer wavelengths, and the hot dust that dominates the $H K$ bands reacts with delayed brightening to AD flux increases. The time lag between $\mathrm{AD}$ and dust emission can be estimated by the separation of the $z$ - and $K$-band peaks, yielding $\tau \sim 50$ days, 


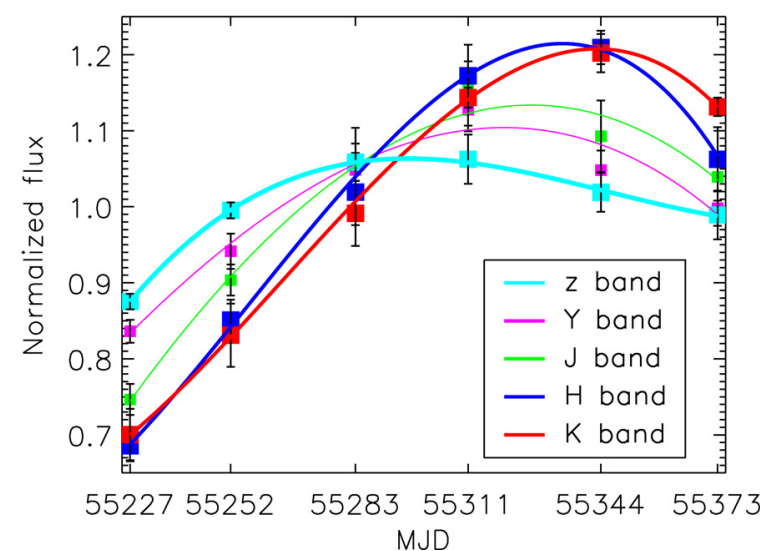

Fig. 2. Calibrated Omega 2000 photometry of NGC 4151. The mean flux in each band is normalized to unity to show the relative variability.

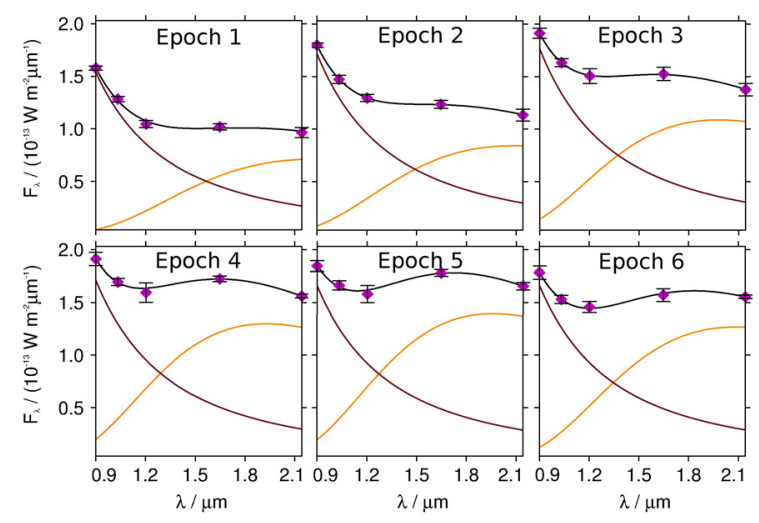

Fig. 3. Two-component decomposition of the SED into AD and hot dust contribution. Magenta squares show our Omega 2000 zYJHK data. Purple and orange lines represent the power-law and blackbody contributions, whereas the black lines show the sum of these two components.

which is consistent with observations by Minezaki et al. (2004) and Hönig \& Kishimoto (2011).

The separate panels in Fig. 3 show the temporal evolution of the blackbody (dust) and power-law (AD) contributions. We observe a significant rise in the hot dust emission from epoch 1 (MJD 55 227) to epoch 5 (MJD 55344), in correspondence to the clear flux increase in the $H$ and $K$ bands.

The hot dust temperature and solid angle are presented in Fig. 4 for the six epochs. We find a temperature increase from roughly $1300 \mathrm{~K}$ to $1500 \mathrm{~K}$, with a peak at MJD 55319, 30 days after the highest AD state (MJD 55289 ). The solid angle decreases from epoch 1 (MJD 55 227) to epoch 3 (MJD 55283 ), followed by a monotonic increase until epoch 6 (MJD 55 373).

\section{Discussion}

Following an increase in the brightness of the AD in NGC 4151, we find a significant rise of the emission of the innermost hot dust, peaking about 50 days after the AD emission peaks. Furthermore, the hot dust temperature rises significantly from $\sim 1300 \mathrm{~K}$ to $1500 \mathrm{~K}$ as a reaction to the increased AD radiation. The hot dust in NGC 4151 is apparently not destroyed following the high AD state around MJD 55289 , but is simply heated up, suggesting that the hot dust is cooler than sublimation temperature. It therefore seems likely that the dust in this galaxy is not located as far in as possible, but is located or formed farther out.

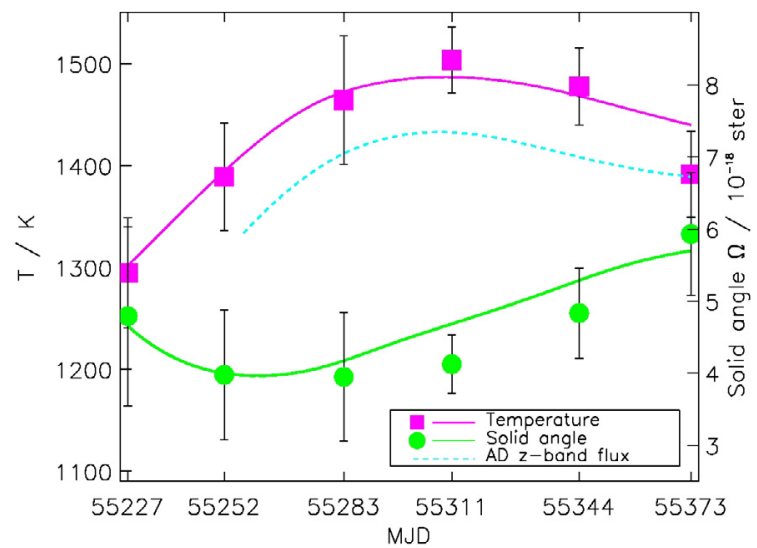

Fig. 4. Temporal evolution of the blackbody temperature and solid angle, derived from the two-component decomposition (magenta squares and green circles). The dashed cyan line shows the $z$-band flux of the $\mathrm{AD}$ in arbitrary units, shifted by 30 days. The solid magenta and green lines represent the temperature and solid angle as calculated from our simplified torus model (see Sect. 5).

We point out, however, that the observed behavior of the temperature as well as the solid angle deviates significantly from what we would expect if the hot dust were well represented by a single blackbody function, i.e., a compact dust distribution with a single temperature. The temperature peaks only $\sim 30$ days after the highest AD state, whereas the $K$-band flux shows a delay of $\sim 50$ days. Moreover, the solid angle decreases from epoch 1 to epoch 3 , before it starts to rise monotonically until epoch 6 .

This observed behavior might be resolved, if we assume a radially extended distribution of the innermost, hot dust. In this case, the dust would be better approximated by a continuous radial distribution of blackbody components with different temperatures where higher temperatures correspond to a smaller distance from the source. To test this assumption, we applied a very simplified torus model making use of the following simplifications:

(1) We approximated the continuous radial structure as a discrete sum of only a few blackbody components at different radial distances from the center. For each radius, we used a single radiating surface, given by a single blackbody function.

(2) Our model was static, i.e. the dust distribution did not vary over time.

(3) Only directly illuminated dust contributed significantly to the observed flux (Hönig \& Kishimoto 2010).

(4) The change of the dust temperature at each radius due to the varying irradiation is given by $\mathrm{d} T / T=1 / 4 \cdot \mathrm{d} L / L$ (Hönig \& Kishimoto 2011), assuming that changes in the incident radiation are entirely reprocessed by the dust. We used the blackbody assumption (absorption efficiency $Q_{\mathrm{abs}}(v, T)=1$ ).

(5) As the main heating source for the dust is the $U V$ part of the $\mathrm{AD}$ radiation, we approximated $\mathrm{d} L / L \approx \mathrm{d} L_{U V} / L_{U V}$. Considering $\Delta F_{\lambda} \propto \lambda^{-2}$ (Meusinger et al. 2011), and assuming $F_{\lambda} \propto \lambda^{-1.75}$ in the $U V-z$ regime (Zheng et al. 1997; Kishimoto et al. 2008), we predicted the $U V$ luminosity variations by the $z$-band variations according to $\mathrm{d} L_{U V} / L_{U V} \approx$ $1.8 \cdot \mathrm{d} L_{z} / L_{z}$.

A model consisting of three blackbody components, with 5\% (by solid angle) of the hot dust located at 25 light days distance from the source, $25 \%$ at 50 light days and $70 \%$ at 100 light days, already matches our data sufficiently well (see Fig. 4). Including 
Table 1. $z Y J H K$ photometric fluxes of the nucleus of NGC 4151, and fit parameters resulting from the two-component decomposition.

\begin{tabular}{|c|c|c|c|c|c|c|c|c|}
\hline \multirow{2}{*}{$\begin{array}{l}\text { Date } \\
(2010)\end{array}$} & \multicolumn{5}{|c|}{$F_{\lambda}\left(10^{-13} W^{-2} \mu \mathrm{m}^{-1}\right)$} & \multirow{2}{*}{$\begin{array}{c}\Omega \\
\left(10^{-18} \text { ster }\right)\end{array}$} & \multirow{2}{*}{$\begin{array}{c}T \\
(\mathrm{~K})\end{array}$} & \multirow{2}{*}{$\begin{array}{c}C \\
\left(10^{-25} \mathrm{Wm}^{-2} \mu \mathrm{m}^{-1}\right)\end{array}$} \\
\hline & $z$ & $Y$ & $J$ & $H$ & $K$ & & & \\
\hline Jan. 30 & $1.58 \pm 0.02$ & $1.28 \pm 0.03$ & $1.05 \pm 0.03$ & $1.02 \pm 0.03$ & $0.97 \pm 0.05$ & $4.79 \pm 1.24$ & $1295 \pm 54$ & $0.94 \pm 0.02$ \\
\hline Feb. 24 & $1.80 \pm 0.02$ & $1.47 \pm 0.04$ & $1.30 \pm 0.04$ & $1.24 \pm 0.04$ & $1.13 \pm 0.06$ & $3.98 \pm 0.90$ & $1389 \pm 53$ & $1.05 \pm 0.02$ \\
\hline Mar. 27 & $1.91 \pm 0.05$ & $1.63 \pm 0.04$ & $1.51 \pm 0.07$ & $1.53 \pm 0.06$ & $1.38 \pm 0.06$ & $3.95 \pm 0.89$ & $1464 \pm 63$ & $1.08 \pm 0.04$ \\
\hline Apr. 25 & $1.91 \pm 0.06$ & $1.70 \pm 0.03$ & $1.60 \pm 0.09$ & $1.73 \pm 0.03$ & $1.56 \pm 0.02$ & $4.13 \pm 0.41$ & $1503 \pm 33$ & $1.05 \pm 0.04$ \\
\hline May. 24 & $1.85 \pm 0.05$ & $1.66 \pm 0.04$ & $1.58 \pm 0.08$ & $1.78 \pm 0.03$ & $1.66 \pm 0.04$ & $4.83 \pm 0.63$ & $1478 \pm 38$ & $1.02 \pm 0.04$ \\
\hline Jun. 26 & $1.78 \pm 0.06$ & $1.53 \pm 0.04$ & $1.46 \pm 0.05$ & $1.57 \pm 0.06$ & $1.55 \pm 0.02$ & $5.93 \pm 0.85$ & $1392 \pm 42$ & $1.02 \pm 0.04$ \\
\hline
\end{tabular}

further components and a finer radial sampling should improve the fit (but was omitted to avoid ambiguities, given the limited number of data points). In contrast, when using one component only, it is not possible to reproduce the observed behavior. This suggests that, in fact, the innermost hot dust might be well-represented by a radially extended distribution rather than one compact component. Thus, a small innermost fraction of the dust sees the increased AD emission first, therefore increasing its temperature and NIR emission prior to the dust farther out. This leads to a stronger weighting of this small fraction of the hottest, innermost dust, and therefore to an early temperature peak and an overall decrease in the emitting surface in the early epochs, before the other surfaces respond as well.

We emphasize that the key fact pointing to such a radially extended dust distribution is the temperature evolution, specifically the result that the temperature peaks significantly earlier than the $H K$-band fluxes. This behavior is also obtained for different values of $\alpha=$ const. (with $1.5 \leq \alpha \leq 2.5$ ), and thus appears to be robust. We note that the observed variation of the solid angle presented in Fig. 4 is not statistically significant within its errors (but a similar behavior is obtained for all $\alpha \in[1.5,2.5]$ ). However, while the evolution of the solid angle alone cannot constrain the dust structure, the shown variation would simply support a radially extended structure as already suggested by the temperature evolution. This extended morhphology is consistent with the observed slight discrepancy between measured NIR dust reverberation radii and NIR interferometric radii for a sample of type 1 AGNs (Kishimoto et al. 2011). Furthermore, it is expected from accretion wind scenarios (see Sect. 1) in which the BLR and the dust torus are assumed to be essentially the same phenomenon, and the transition between BLR and dust torus occurs simply when the dust condensation conditions are fulfilled.

\section{Conclusions}

In this project, we use dust reverberation mapping as a tool to explore the dust morphology in AGNs. As a first step, we carried out NIR Omega 2000 multi-band photometry of the nucleus of NGC 4151, in six epochs in 2010, supplemented by a NIR IRTF spectrum in 2010 February. We present here our first results:

- From our NGC 4151 NIR light curves, we find a lag time of $\sim 50$ days between AD emission (represented by the $z$ band) and $K$-band hot dust emission, in very good agreement with previously measured $K$-band hot dust reverberation lags.

- We observe a significant rise in the hot dust emission following times of high AD brightness. Moreover, the hot dust blackbody temperature increased as a reaction to the enhanced irradiation, with a lag time of roughly 30 days.

- Despite the increased AD brightness, we find indications that the distribution of the innermost dust is static and without significant dust destruction or reformation in the observed period. This is consistent with Pott et al. (2010), but in contrast to the analysis of Koshida et al. (2009). Furthermore, the dust morphology does not seem to have a sharp inner edge, but appears to be radially extended.

Our central finding is that a stationary dust distribution can explain the observed flux and temperature variations in NGC 4151. While changes in dust morphology are not expected on half-year timescales $^{2}$, for NGC 4151 we can exclude significant dust destruction in response to the observed AD variation. We emphasize that we do not see explicit signatures of intrinsic dust formation in our current data. With our ongoing observations of NGC 4151 with Omega 2000, however, we will obtain a longer time series that might allow us, in combination with a radiative transfer model, to detect a potential change in the dust morphology. To test the generality of our results, we are monitoring six additional bright and variable Seyfert 1 AGNs with the GROND camera (grizJHK bands, ESO La Silla), and in the optical with the All-Sky Automated Survey for Supernovae (PI: K. Stanek).

Acknowledgements. We are very thankful to the anonymous referee for significantly improving this manuscript. We thank R. van Boekel for providing a database of main sequence star atmospheres. K. S. acknowledges support by "IMPRS for Astronomy \& Cosmic Physics at the University of Heidelberg". B. M. P. is grateful for the support of the US NSF through grant AST-1008882.

\section{References}

Antonucci, R. 1993, ARA\&A, 31, 473

Barvainis, R. 1987, ApJ, 320, 537

Cushing, M. C., Vacca, W. D., \& Rayner, J. T. 2004, PASP, 116, 362 Elitzur, M., \& Shlosman, I. 2006, ApJ, 648, L101

Elvis, M., Marengo, M., \& Karovska, M. 2002, ApJ, 567, L107

Heisler, C. A., Lumsden, S. L., \& Bailey, J. A. 1997, Nature, 385, 700

Hönig, S. F., \& Kishimoto, M. 2010, A\&A, 523, A27

Hönig, S. F., \& Kishimoto, M. 2011, A\&A, 534, A121

Kishimoto, M., Antonucci, R., Blaes, O., et al. 2008, Nature, 454, 492

Kishimoto, M., Hönig, S. F., Antonucci, R., et al. 2011, A\&A, 527, A121

Kobayashi, Y., Sato, S., Yamashita, et al. 1993, ApJ, 404, 94

Koshida, S., Yoshii, Y., Kobayashi, Y., et al. 2009, ApJ, 700, L109

Kovács, Z., Mall, U., Bizenberger, et al. 2004, SPIE, 5499, 432

Krolik, J. H., \& Begelman, M. C. 1988, ApJ, 329, 702

Meusinger, H., Hinze, A., \& de Hoon, A. 2011, A\&A, 525, A37

Minezaki, T., Yoshii, Y., Kobayashi, Y., et al. 2004, ApJ, 600, L35

Osterbrock, D. E., \& Ferland, G. J. 2006, Astrophysics of gaseous nebulae and active galactic nuclei (Sausalito: University science books)

Peng, C. Y., Ho, L. C., Impey, C. D., \& Rix, H.-W. 2002, AJ, 124, 266

Pott, J.-U., Malkan, M. A., Elitzur, M., et al. 2010, ApJ, 715, 736

Rayner, J. T., Toomey, D. W., Onaka, P. M., et al. 2003, PASP, 115, 362

Riffel, R. A., Storchi-Bergmann, T., \& McGregor, P. J. 2009, ApJ, 698, 1767

Schartmann, M., Burkert, A., Krause, M., et al. 2010, MNRAS, 403, 1801

Skrutskie, M. F., Cutri, R. M., Stiening, R., et al. 2006, AJ, 131, 1163

Swain, M., Vasisht, G., Akeson, R., et al. 2003, ApJ, 596, L163

Urry, C. M., \& Padovani, P. 1995, PASP, 107, 803

Vacca, W. D., Cushing, M. C., \& Rayner, J. T. 2003, PASP, 115, 389

Weigelt, G., Hofmann, K.-H., Kishimoto, M., et al. 2012, A\&A, 541, L9

Zheng, W., Kriss, G. A., Telfer, et al. 1997, ApJ, 475, 469

2 Since the BLR clouds move with speeds of $v \ll c$ (Elvis et al. (2002): $v \gtrsim 1000 \mathrm{~km} \mathrm{~s}^{-1}$ ), the time scale for changes in the dust distribution is much larger than our six months of monitoring. 